\title{
Mutation-in-Brief
}

\section{A Novel A461S Mutation of PTPN11 in a Female with LEOPARD Syndrome}

\author{
Rie Yoshida ${ }^{1}$ and Tsutomu Ogata ${ }^{1}$ \\ ${ }^{I}$ Department of Endocrinology and Metabolism, National Research Institute for Child Health and \\ Development, Tokyo, Japan
}

\section{Introduction}

LEOPARD syndrome (LS) is a congenital developmental disorder and is an acronym for multiple lentigines, electrocardiographic conduction abnormalities, ocular hypertelorism, pulmonary stenosis, abnormalities of genitalia, retardation of growth, and sensorineural deafness (1). These clinical features overlap those of Noonan syndrome (NS), and heterozygous germline PTPN11 mutations have been identified in approximately $45 \%$ of NS patients and in $>80 \%$ of LS patients (1). Herein, we report a novel mutation of PTPN11 in a female with LS.

\section{Patient Report}

A 34-year-old Japanese female was referred to us for molecular diagnosis of LS. She had numerous nevi, ocular hypertelorism, cardiac diseases (mitral valve insufficiency and right bundle branch block with left axis deviation) and sensorineural deafness. Her height was $160 \mathrm{~cm}$ (+0.4 SD), and her weight was $62 \mathrm{~kg}$ (+1.0 SD). This study was approved by the ethical committee of our institution. After obtaining informed

Received: August 21, 2008

Accepted: September 18, 2008

Correspondence: Dr. Rie Yoshida, Department of Endocrinology and Metabolism, National Research Institute for Child Health and Development, 2-10-1, Ohkura, Setagaya-ku, Tokyo 157-8535, Japan

E-mail: ries@nch.go.jp consent, direct sequencing was performed for PTPN11 using leukocyte genomic DNA from this patient in accordance with previously reported methods (2). Consequently, a novel heterozygous c.1381G>T (p.A461S) mutation was identified on exon 12 (Fig. 1).

\section{Discussion}

We identified a novel mutation (c. $1381 \mathrm{G}>\mathrm{T}$, p.A461S) of PTPN11 in a female with LS. Recent
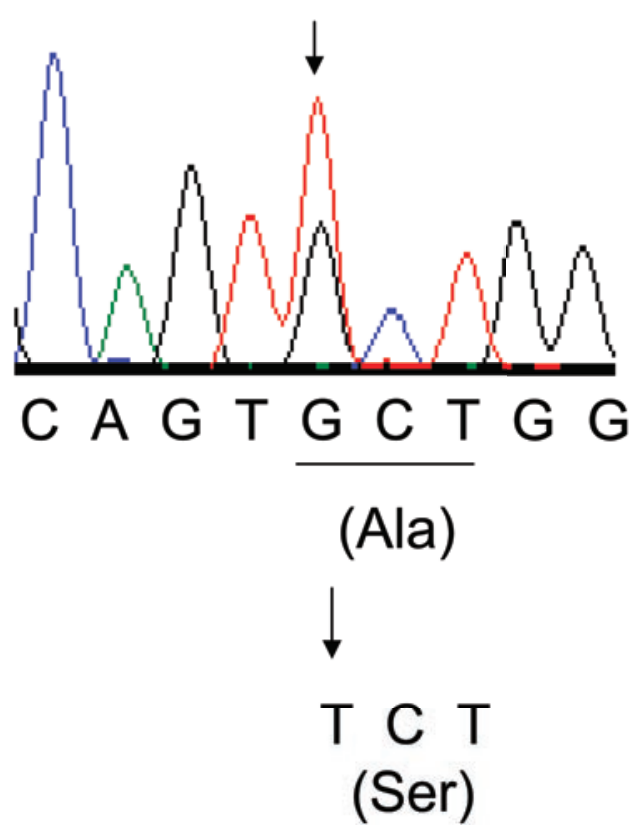

Fig. 1. An electrochromatogram showing a heterozygous c.1381G>T (p.A461S) mutation (an arrow). 


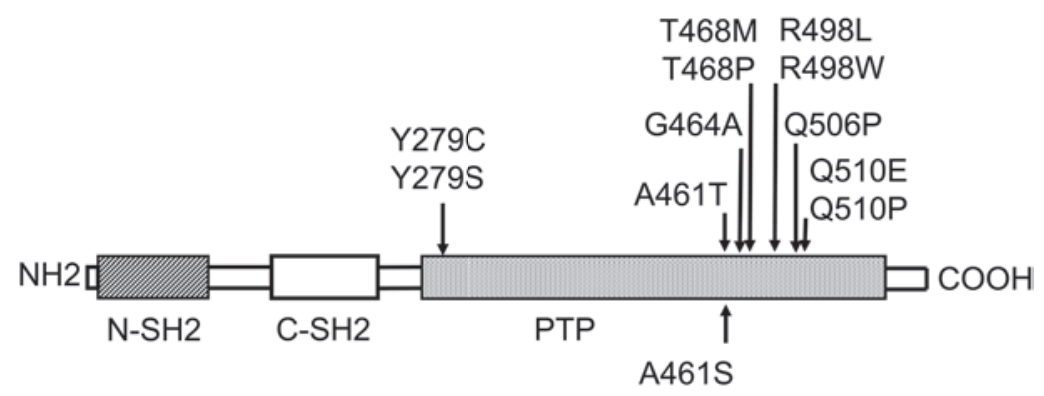

Fig. 2. Schematic representation of PTPN11 protein showing the distribution of mutations reported in LS (above). The position of the mutation detected in this study is also indicated (below). N-SH2 and C-SH2 are the N-terminal and C-terminal tandemly arranged SH2 domains and are followed by a protein PTP domain at the C-terminus.

studies have shown that LS-associated PTPN11 mutants impair catalytic functions and exert dominant negative effects, whereas NS-associated PTPN11 mutants exert gain-of-function effects with excessive phosphatase activities $(1,3)$. Indeed, the mutations in LS and NS are mutually exclusive, and all eleven types of mutation in LS (two recurrent Y279C and T468M mutations and additional Y279S, A461T, T468P, G464A, R498L, R498W, Q506P, Q510E and Q510P mutations) have been identified at the catalytic cleft of the PTPN11 protein (1, 3-5) (Fig. 2). Since the novel c.1381G $>$ T (p.A461S) mutation in this patient also resides at the catalytic cleft, this is consistent with the positional properties of PTPN11 mutations leading to LS.

\section{Acknowledgements}

This work was supported in part by grants for Child Health and Development (17C-2) and for Research on Children and Families (18-005) from the Ministry of Health, Labor and Welfare of Japan.

\section{References}

1. Gelb BD, Tartaglia M. Noonan syndrome and related disorders: dysregulated RAS-mitogen activated protein kinase signal transduction. Hum Mol Genet 2006;15:R220-6.

2. Yoshida R, Hasegawa T, Hasegawa Y, Nagai T, Kinoshita E, Tanaka Y, et al. Protein tyrosine phosphatase, nonreceptor type 11 mutation analysis and clinical assessment in 45 patients with Noonan syndrome. J Clin Endocrinol Metab 2004;89:3359-64.

3. Kontaridis MI, Swanson KD, David FS, Barford D, Neel BG. PTPN11 (Shp2) mutations in LEOPARD syndrome have dominant negative, not activating, effects. J Biol Chem 2006;281:678592.

4. Digilio MC, Sarkozy A, Pacileo G, Limongelli G, Marino B, Dallapiccola B. PTPN11 gene mutations: linking the Gln510Glu mutation to the "LEOPARD syndrome phenotype". Eur J Pediatr 2006;165:803-5.

5. Seishima M, Mizutani Y, Shibuya Y, Arakawa C, Yoshida R, Ogata T. Malignant melanoma in a woman with LEOPARD syndrome: identification of a germline PTPN11 mutation and a somatic BRAF mutation. Br J Dermatol 2007;157:12979. 